\title{
Astrometric observations of inner Saturnian satellites
}

\author{
F. Poulet ${ }^{1}$ and B. Sicardy ${ }^{2}$ \\ 1 NASA Ames Research Center, MS 245/3, Moffett Field, CA 94035, USA \\ e-mail: poulet@cosmic.arc.nasa.gov \\ 2 Observatoire de Paris, Institut Universitaire de France, 92195 Meudon Cedex Principal, France \\ e-mail: sicardy@obspm.fr
}

Received 31 August 2000 / Accepted 24 October 2000

\begin{abstract}
This paper presents observations of four inner Saturnian satellites $(\mathrm{SX}=$ Janus, SXI $=$ Epimetheus, SXVI = Prometheus and SXVII = Pandora) made in August and November 1995 during the Earth and Sun crossings of Saturn's ring plane, respectively. The August 1995 data combine data taken with the Adonis optics system mounted on the ESO 3.6-m telescope at La Silla, and images from the Hubble Space telescope (HST). The November 1995 data are based on HST images only. Orbits fits show that these observations combined with those of Nicholson et al. (1996) have residuals of about 0.05 arcsec.
\end{abstract}

Key words. planets and satellites: individual: Saturn - astrometry

\section{Introduction}

Several campaigns of observations of Saturn's satellites have been done during the last years in order to prepare the future Cassini mission and to improve the contemporary theories given by Duriez \& Vienne (1991), Vienne \& Duriez (1991, 1992), Dourneau (1993) and Harper \& Taylor (1993). However, these new data concerned only the Saturnian major satellites (Harper et al. 1997, 1999; Qiao et al. 1999). The 6 Saturn's inner satellites smaller than Mimas (radius $200 \mathrm{~km}$ ) are much more difficult to detect from the Earth because they orbit close to Saturn's bright rings, at distances ranging from 2.2 to 2.5 Saturn's radii, with radii of only $15-89 \mathrm{~km}$. The small satellites were all discovered either during the ring plane crossings ("RPXs" for short) in 1966 and 1980 or during the Voyager spacecraft encounters in November 1980 (Voyager 1) and August 1981 (Voyager 2). Except for SX and SXI, they were not observed again until the last RPXs by the Earth and the Sun in 1995. These particular configurations provided a rare opportunity to detect much easily small objects near the rings, since the scattered light from the rings was then sufficiently reduced to permit their detection. With the coming of the Cassini mission and the need of dynamic predictions of Saturn's satellites to be based on precise astrometric observations, it is becoming more and more important to collect high quality observations for these small objects.

In this paper, we publish the positions of four inner Saturnian satellites measured during our campaign of observations of RPXs in August and November 1995.

Send offprint requests to: F. Poulet
We then combine previous HST results obtained by Nicholson et al. (1996) with our new measurements in order to check for consistency and for potential systematic errors of these sets of data.

\section{Observations and data reduction}

\subsection{Ground-based observations set}

During two nights (9 and 10 August 1995) close to the Earth RPX, ground-based observations in the nearinfrared were carried out with the Adonis adaptive optics system, mounted on the ESO 3.6-m telescope at La Silla, Chile. The conditions of observations are detailed in Poulet et al. (2000a). In spite of regular seeing conditions, the adaptive optics system reduces the FWHM of the point spread function to about 0.5 arcsec or less.

Standard infrared data reduction procedures were applied to each individual ESO frame: flat-fielding to correct pixel-to-pixel sensivity variations, sky subtraction and cosmic ray elimination. A correction for specific detector electronic periodic patterns was also performed.

\subsection{Hubble space telescope sets}

The HST Wide Field Planetary Camera 2 (hereafter, WFPC2) data, downloaded from HST data archive, are grouped in three main sets: eight images taken on August 6, eleven images taken on November 17 and eight images taken on November 18. The first two sets result from an observing program by $M$. Tomasko and E. Karkoschka [program ID 6030], the following set has been obtained by $J$. Caldwell [ID 6328]. 
Table 1. Number of detections in August 1995

\begin{tabular}{llll}
\hline & SX & SXVI & SXVII \\
\hline $06 / 08 / 1995$ & 1 & 5 & 6 \\
$09 / 08 / 1995$ & 7 & 0 & 4 \\
$10 / 08 / 1995$ & 10 & 0 & 6 \\
$10 / 08 / 1995^{*}$ & 7 & 12 & 15 \\
\hline
\end{tabular}

* Data set of Nicholson et al. (1996).

Table 2. Number of detections in November 1995

\begin{tabular}{lllll}
\hline & SX & SXI & SXVI & SXVII \\
\hline $17 / 11 / 1995$ & 2 & 7 & 2 & 11 \\
$18 / 11 / 1995$ & 8 & 8 & 5 & 0 \\
$21 / 11 / 1995^{*}$ & 8 & 6 & 8 & 7 \\
\hline
\end{tabular}

* Data set of Nicholson et al. (1996).

The HST images were calibrated frames provided by the Space Telescope Science Institute. Classical flatfielding and dark subtraction were performed during HST pipeline processing (Biretta et al. 1996). We took into account optical distorsion in the HST planetary camera (Holtzman et al. 1995).

\section{Results}

\subsection{Detections and astrometric measurements}

Our various detections are summarized in Tables 1 and 2 and compared with the number of detections obtained by Nicholson et al. (1996) during the RPXs of 10 August and 21 November 1995. In August, SX and SXVII are identified both on ESO and HST images. SXVI is detected on HST images only, and lags the expected longitudinal position by about 19 degrees. Our data sets increase significantly the number of detections of these satellites.

The strong local gradient of light due to the planet can systematically bias the measurements of the object positions. We experimented with several methods of subtracting the scattered light from the planet. For the August results (ESO and HST images), the background is determined from a robust, low order, polynomial fit to each line parallel to the ring plane. For the November results, we have measured the scattered light of the planet on a portion of a circle centered on Saturn, and going through the pixel under study. Typically, this arc of circle extends angularly between 5 and 15 degrees away from the studied point. Then, we calculated the medians of the scattered light on that arc and we removed the resulting value from the pixel.

When available, a known satellite was used as an astrometric reference point in each image. Ephemerides of the satellites were taken from the on line software at the Planetary Data System's Rings Node (Showalter 1995), which uses ephemeris files provided by the Jet Propulsion Laboratory. For the ESO images, the location of Cassini Division is used as a pointing reference in several images for which reference moons were unavailable. For the HST
Table 3. Planetocentric measurement in August 1995

\begin{tabular}{ccr}
\hline Satellite & $\begin{array}{c}\text { Decimal day } \\
(\mathrm{UT})\end{array}$ & $\begin{array}{r}\text { Planetocentric } \\
\text { positions } \\
\text { (arcsec) }\end{array}$ \\
\hline SX & 6.540820 & 22.54 \\
SX & 9.276620 & 18.36 \\
SX & 9.277315 & 18.42 \\
SX & 9.278009 & 18.57 \\
SX & 9.278704 & 18.61 \\
SX & 9.280092 & 18.66 \\
SX & 9.280787 & 18.79 \\
SX & 9.281488 & 18.82 \\
SX & 10.29687 & -15.22 \\
SX & 10.29965 & -15.69 \\
SX & 10.30312 & -16.24 \\
SX & 10.41542 & -23.44 \\
SX & 10.41611 & -23.39 \\
SX & 10.41680 & -23.35 \\
SX & 10.41819 & -23.35 \\
SX & 10.42028 & -23.26 \\
SX & 10.42097 & -23.19 \\
SX & 10.42167 & -23.18 \\
\hline SXVI & 6.549896 & 21.23 \\
SXVI & 6.552361 & 21.08 \\
SXVI & 6.555220 & 20.91 \\
SXVI & 6.557257 & 20.74 \\
SXVI & 6.559792 & 20.50 \\
\hline SXVII & 6.549896 & -21.69 \\
SXVII & 6.552361 & -21.62 \\
SXVII & 6.555220 & -21.48 \\
SXVII & 6.557257 & -21.38 \\
SXVII & 6.559792 & -21.23 \\
SXVII & 6.565590 & -20.84 \\
SXVII & 9.278021 & 15.32 \\
SXVII & 9.278715 & 15.37 \\
SXVII & 9.280104 & 15.67 \\
SXVII & 9.280799 & 15.78 \\
SXVII & 10.30312 & -22.14 \\
SXVII & 10.39398 & -13.54 \\
SXVII & 10.39468 & -13.35 \\
SXVII & 10.39537 & -13.18 \\
SXVII & 10.39606 & \\
SXVII & 10.39676 & \\
\hline & & \\
\hline
\end{tabular}

* The minus sign means east ansa. See text for the uncertainties in the measurements.

images in which no known satellite is available, Saturn's limb was used as a pointing reference. The rings can then define the north-south location of Saturn's center.

The center of the sources was determined by a centering routine for the brighter satellites, and by picking "by eye" the brightest pixel for the fainter ones. Because of the almost edge-on aspect of the orbits, the pixel locations were then converted to distances from Saturn, as projected onto the plane of the sky. The planetocentric positions are given in Tables $3-5$.

\subsection{Uncertainties}

The uncertainties assigned to the measurements depend on the number of individual measurements combined in 
Table 4. Planetocentric measurements in November 1995

\begin{tabular}{ccr}
\hline Satellite & $\begin{array}{c}\text { Decimal day } \\
(\mathrm{UT})\end{array}$ & $\begin{array}{r}\text { Planetocentric positions* } \\
\text { (arcsec) }\end{array}$ \\
\hline SX & 17.282963 & 15.44 \\
SX & 17.284271 & 15.66 \\
SX & 18.356319 & -19.11 \\
SX & 18.358541 & -19.42 \\
SX & 18.363415 & -19.83 \\
SX & 18.366945 & -20.23 \\
SX & 18.368935 & -20.38 \\
SX & 18.373182 & -20.75 \\
SX & 18.380972 & -21.30 \\
SX & 18.382847 & -21.49 \\
\hline SXI & 17.349953 & -19.85 \\
SXI & 17.352501 & -20.10 \\
SXI & 17.354479 & -20.30 \\
SXI & 17.356516 & -20.48 \\
SXI & 17.359386 & -20.73 \\
SXI & 17.361423 & -20.90 \\
SXI & 17.363958 & -21.11 \\
SXI & 18.356319 & 15.17 \\
SXI & 18.358541 & 15.62 \\
SXI & 18.363415 & 16.07 \\
SXI & 18.366945 & 16.82 \\
SXI & 18.368935 & 17.12 \\
SXI & 18.373182 & 17.57 \\
SXI & 18.380972 & 18.51 \\
SXI & 18.382847 & 18.63 \\
\hline & &
\end{tabular}

* The minus sign means east ansa. See text for the uncertainties in the measurements.

each point, the uncertainties in these measurements, and systematic errors in the pointing reference. For the HST data, we have compared the determination of Saturn's center using a satellite and using the limb of the planet. Both methods agree to within \pm 0.04 arcsec for PC images (0.9 pixel), and \pm 0.07 arcsec for WF images ( 0.7 pixel). So, taking into account the uncertainty in the center of the objects, we assign uncertainties of \pm 0.07 arcsec for the PC data, vs. \pm 0.12 arcsec for the WF images.

The August 1995 profiles of rings seen almost by edgeon extend to the location of the F ring located at 140, $200 \mathrm{~km}$ from the Saturn's center (Poulet et al. 2000b). Comparisons of various ESO ring profiles allow us to estimate uncertainties of $\pm 0.1 \operatorname{arcsec}(\sim 2$ pixels $)$ for ESO measurements.

\subsection{Analysis of the observations}

In this section, we present the orbital elements that were determined by analysis of our sets of observations (Tables 3-5) combined with the positions given by Nicholson et al. (1996) from their HST images taken on 10 August and 20 November 1995. These fits are mainly
Table 5. Planetocentric measurements in November 1995

\begin{tabular}{ccr}
\hline Satellite & $\begin{array}{c}\text { Decimal day } \\
\text { (UT) }\end{array}$ & $\begin{array}{r}\text { Planetocentric positions } \\
\text { (arcsec) }\end{array}$ \\
\hline SXVI & 17.219757 & -20.21 \\
SXVI & 17.222292 & -20.06 \\
SXVI & 18.356319 & -16.89 \\
SXVI & 18.358541 & -17.19 \\
SXVI & 18.366945 & -18.14 \\
SXVI & 18.373182 & -18.82 \\
SXVI & 18.380972 & -19.45 \\
\hline SXVII & 17.219757 & 16.66 \\
SXVII & 17.222292 & 16.98 \\
SXVII & 17.282963 & 21.37 \\
SXVII & 17.284271 & 21.37 \\
SXVII & 17.349953 & 17.48 \\
SXVII & 17.352501 & 17.16 \\
SXVII & 17.354479 & 16.90 \\
SXVII & 17.356516 & 16.63 \\
SXVII & 17.359386 & 16.23 \\
SXVII & 17.361423 & 15.94 \\
SXVII & 17.363958 & 15.59 \\
\hline
\end{tabular}

* The minus sign means east ansa. See text for the uncertainties in the measurements.

Table 6. Orbital elements from August data (longitude is on August 10.5 TDT at Saturn)

\begin{tabular}{llll}
\hline Parameters & SX & SXVI & SXVII \\
\hline$a(\mathrm{~km})$ & $151550 \pm 80$ & $139310 \pm 80$ & $141742 \pm 100$ \\
$n\left({ }^{\circ} /\right.$ day $)$ & $517.8 \pm 0.4$ & $587.7 \pm 0.4$ & $572.6 \pm 0.6$ \\
$\lambda_{0}\left(^{\circ}\right)$ & $34.58 \pm 0.20$ & $339.44 \pm 0.15$ & $96.53 \pm 0.25$ \\
$e^{*}$ & 0.0066 & 0.0024 & 0.0042 \\
$\varpi^{*}\left(^{\circ}\right)$ & 108.0 & 236.4 & 6.9 \\
$\Delta \lambda_{0}{ }^{* *}\left({ }^{\circ}\right)$ & -0.67 & -18.53 & 0.29 \\
rms (arcsec) & 0.053 & 0.050 & 0.070 \\
\hline
\end{tabular}

* Assumed.

** $(\mathrm{O}-\mathrm{C})$ of $\lambda_{0}$.

performed in order to check for consistency and for potential systematic errors. The fitted parameters are the orbital radius $a$, the mean motion $n$ and the longitude $\lambda_{0}$ measured from the ascending node of Saturn's equatorial plane on the Earth's equatorial plane J2000 at reference time $t_{0}$ (defined to be 10 August 1995, 12:00 TDT at Saturn for the August data, and 21 November 1995, 12:00 TDT at Saturn for the November data). The eccentricity $e$ and the longitude of pericentre $\varpi$ have been given by Yoder et al. (1989) and Nicholson et al. (1992) for the coorbital satellites, and Yoder et al. (1989) and Jacobson (1995) for the shepherds. Tables 6 and 7 give the three derived orbital elements and the difference $\Delta \lambda_{0}$ between the expected longitude and the observed longitude at epoch.

We have calculated the postsolution root-meansquare residuals of the observations. The rms residuals 
Table 7. Orbital elements from November data (longitude is on November 21.5 TDT at Saturn)

\begin{tabular}{lllll}
\hline Parameters & SX & SXI & SXVI & SVXI \\
\hline$a(\mathrm{~km})$ & $151470 \pm 50$ & $151425 \pm 100$ & $139395 \pm 30$ & $141720 \pm 30$ \\
$n\left({ }^{\circ} /\right.$ day $)$ & $518.2 \pm 0.3$ & $518.4 \pm 0.6$ & $587.2 \pm 0.2$ & $572.7 \pm 0.2$ \\
$\lambda_{0}\left(^{\circ}\right)$ & $134.25 \pm 0.15$ & $298.61 \pm 0.30$ & $349.96 \pm 0.10$ & $52.70 \pm 0.10$ \\
$e^{*}$ & 0.0066 & 0.0126 & 0.0024 & 0.0042 \\
$\varpi^{*}\left(^{\circ}\right)$ & 319.0 & 74.0 & 160.4 & 274.70 \\
$\Delta \lambda_{0}{ }^{* *}\left({ }^{\circ}\right)$ & 0.41 & 0.58 & -18.79 & -0.81 \\
rms (arcsec) & 0.041 & 0.072 & 0.021 & 0.021 \\
\hline
\end{tabular}

* Assumed, ${ }^{* *}(\mathrm{O}-\mathrm{C})$ of $\lambda_{0}$.

$(\sim 0.05$ arcsec $)$ for all the points are comparable to the errors in the positions measurements indicating that the data are consistent.

\section{Conclusion}

These observations made during the ring plane crossings in August and November 1995 are a significant part of scarce observations of small inner satellites of Saturn. New observations of these satellites with Hubble Space Telescope are ongoing (French et al. 1998), but the extreme difficulty to obtain observating time with this instrument should lead to the community of observers of Saturn's satellites to apply observating time with performant instruments using the Adaptive optics.

Acknowledgements. We thank P. D. Nicholson, E. Karkoschka for their involvement in the HST observations and useful discussions. A part of this work was performed while F. Poulet held a National Research Council - NASA ARC Research Associateship.

\section{References}

Biretta, J. A., Burrows, C., Holtzman, J., et al. 1996, WFPC2 Instrument Handbook, Version 4.0, Space Telescope Science Inst., Baltimore, MD
Dourneau, G. 1993, A\&A, 267, 292

Duriez, L., \& Vienne, A. 1991, A\&A, 243, 263

French, R. G., Hall, K. J., McGhee, C. A., et al. 1998, BAAS, 30, 0204

Harper, D., \& Taylor, D. B. 1993, A\&A, 268, 326

Harper, D., Murray, C. D., Beurle, K., et al. 1997, A\&AS, 121, 65

Harper, D., Beurle, K., Williams, I. P., et al. 1999, A\&AS, 136, 257

Jacobson, R. A. 1995, BAAS, 28, 1185

Holtzman, J. A., Hester, J., Casertano, et al. 1995, PASP, 107, 156

Nicholson, P. D., Hamilton, D. P., Matthews, K., \& Yoder, C. F. 1992, Icarus, 100, 464

Nicholson, P. D., Showalter, M. R., Dones, L., et al. 1996, Science, 272, 509

Poulet, F., Sicardy, B., Nicholson, P. D., Karkoschka, E., \& Caldwell, J. 2000a, Icarus, 144, 135

Poulet, F., Sicardy, B., Dumas, C., Jorda, L., \& Tiphene, D. 2000b, Icarus, 145,147

Qiao, R., Shen, K., Liu, J., \& Harper, D. 1999, A\&AS, 137, 1

Showalter, M. R. 1995, Saturn Viewer software at the Planetary Data System's Ring Node, NASA Ames Research Center

Vienne, A., \& Duriez, L. 1991, A\&A, 246, 619

Vienne, A., \& Duriez, L. 1992, A\&A, 257, 351

Yoder, C. F., Synott, S. P., \& Salo, H. 1989, AJ, 98, 1875 